L'activité protéolytique totale diminue légèrement au cours de la conservation mais, par contre l'activité protéolytique libre nulle dans la viande frâ̂che atteint $30 \mathrm{p}$. Ioo de l'activité totale.

La lipolyse et l'oxydation des gras sont pratiquement nulles.

\author{
SUMMARY \\ EVOLUTION OF SOME PHYSICO-CHEMICAL CHARACTERISTICS \\ IN DEEP FROZEN PIG MUSCLE PRESERVED AT $-20^{\circ} \mathrm{C}$
}

The object of this report is to study the physico-chemical modifications of pig muscle constituents as affected by deep freezing and preservation in a deep frozen state. Samples of the Longissimus Dorsi of pigs weighing $100 \mathrm{~kg}$ were deep frozen in plastic bags at $-20^{\circ} \mathrm{C}$ in a vacuum. They were thawed after 4 and 8 months of preservation. The following observations may be made from the data gathered :

The amount of dry matter slightly increases.

Water retention power is diminished.

There is no protein degradation during storage.

There is no significant deamination.

Nucleotide dephosphorylation during 8 months storage is analogous to that wich occurs in fresh meat after 8 days at $+4^{\circ} \mathrm{C}$.

Total proteolytic activity decreases slightly during preservation but free proteolytic activity (non existent in fresh meat) reaches $30 \mathrm{p}$. Ioo of the total activity.

Lipolysis and oxydation of fats are almost non existent.

\title{
INFLUENCE DU MODE DE CONGÉLATION ET DE DÉCONGÉLATION DES JAMBONS SUR LES RENIEMENTS APHÈ SAUMURAgE ET CUISSON
}

\author{
J. CHARPENTIER et B. JACQUET \\ Station de Recherches sur la Viande, \\ Centre de Recherches de Clermont-Ferrand, 63 -Saint-Genès-Champanelle \\ Institut national de la Recherche agronomique \\ Laboratoire de Charcuterie expérimentale, \\ Centre technique de Salaison et de Charcuterie comestible, 78 -Jouy-en-Josas
}

\section{RÉSUMÉ}

Cette expérience avait pour but de préciser l'influence de la vitesse de congélation et de décongélation sur le rendement technologique du jambon de Paris après décongélation, saumurage et cuisson. Les résultats obtenus montrent que les pertes à la décongélation sont très variables. 
Elles atteignent au maximum 6,5 p. Ioo du poids du jambon. Lorsque le pH musculaire est relativement élevé, les pertes à la décongélation sont faibles. L'analyse statistique ne permet pas de mettre en évidence une influence hautement significative du mode de congélation ou de décongélation sur l'importance des pertes à la décongélation. L'association de la congélation lente à la décongélation rapide tend toutefois à entraîner une exsudation plus abondante. Dans le cas de congélation et de décongélation lentes, les pertes à la cuisson ont tendance à augmenter et par voie de conséquence, le rendement technologique diminue. Toutefois, l'influence du $\mathrm{pH}$ musculaire reste prépondérante puisque les valeurs élevées du $\mathrm{pH}$ conduisent systématiquement à de hauts rendements quel que soit le mode de congélation ou de décongélation utilisé. Il semblerait, en outre, que la congélation rapide confère aux produits finis une meilleure acceptabilité.

\title{
SUMMARY
}

\section{EFFECTS OF HAM DEEP-FREEZE AND THAWING METHODS ON YIELD AFTER SALTING AND COOKING}

The object of this experiment was to determine the effects of the speed of deep-freezing and thawing on the technological yield of Paris ham after thawing, salting and cooking. Results obtained show that loss at thawing is very variable. It reaches at most 6.5 p. Ioo of ham weight. When the muscle $\mathrm{pH}$ is relatively high, thawing losses are low. Statistic analysis does not show that deep-freezing or thawing methods have a highly significant effect on thawing loss. However, the association of slow deep-freezing with rapid thawing tends to cause more sweating. As far as slow deep-freezing and thawing are concerned, cooking losses increase and, consequently, the technological yield diminishes. However, the effect of muscular $\mathrm{pH}$ remains important since high $\mathrm{pH}$ values systematically produce high yields, no matter what the method of deep-freezing or thawing. It appears that rapid freezing makes the finished product more acceptable.

\section{RECONSTITUTION DU GLYCOGẼNE MUSCULAIRE ET HÉPATIQUE A L'ISSUE D'UNE DÉPLÉTION PROVOQUÉ PAR UNE INJECTION INTRAVEINEUSE D'ADRENALINE}

\author{
J. CHARPENTIER et G. MONIN \\ Station de Recherches sur la Viande, \\ Centre de Recherches de Clermont-Ferrand, 63 -Saint-Genès-Champanelle \\ Institut national de la Recherche agronomique
}

\section{RÉSUMÉ}

Nous avons au cours de cette expérience déterminé le délai de resynthèse du glycogène hépatique et musculaire à l'issue d'une déplétion provoquée par l'injection d'adrénaline chez 28 porcs Large White de go à I Io kg. La connaissance de ce délai présente un grand intérêt puisque le taux 